\title{
Upper and Lower Bounds for Generalized Wiener Indices on Unicyclic Graphs
}

\author{
Álvaro Martínez-Pérez ${ }^{a, *}$, José M. Rodríguez ${ }^{b}$ \\ ${ }^{a}$ Departamento de Análisis Económico y Finanzas, Universidad de \\ Castilla-La Mancha. Avda. Real Fábrica de Seda, s/n. 45600 Talavera \\ de la Reina, Toledo, Spain. \\ ${ }^{b}$ Departamento de Matemáticas, Universidad Carlos III de Madrid, \\ Avenida de la Universidad 30, 28911 Leganés, Madrid, Spain. \\ alvaro.martinezperez@uclm.es, jomaro@math.uc3m.es
}

(Received January 13, 2022)

\begin{abstract}
The aim of this paper is to obtain new inequalities for a large family of generalizations of the Wiener Index and to characterize the set of extremal graphs with respect to them. Our main results provide upper and lower bounds for these topological indices on unicyclic graphs.
\end{abstract}

\section{Introduction}

A topological descriptor is a single number that it is computed on the molecular graph of a compound and represents some chemical structure in terms of this graph. These descriptors are common and relevant in the field of mathematical chemistry and especially in the QSPR/QSAR investigations.

When a topological descriptor correlates with a molecular property of certain chemical compounds, then it is called a topological index. Thus,

\footnotetext{
${ }^{*}$ Corresponding author.
} 
topological indices capture some essential physicochemical property into a single number and this can be used to analyze those properties. This can be very interesting for practitioners. For example, although only about 1000 benzenoid hydrocarbons are known, the number of theoretically possible benzenoid hydrocarbons is huge. If we consider, for instance, the number of possible benzenoid hydrocarbons with 35 benzene rings, it is $5.85 \times$ $10^{21}$ [28]. Therefore, a good model capable of predicting physico-chemical properties of currently unknown species is extremely useful.

The main reason for the use of topological indices is to obtain predictions of some property of certain molecules (see, e.g., $[9,13,15,26]$ ). Whenever a topological descriptor shows a better correlation for some property on some group of chemical compounds a new topological index appears. This way hundreds of topological indices have been defined and studied, starting with the seminal work by Wiener, [31], who found a correlation between his index and paraffin boiling points.

The Wiener index of $G$ is defined as

$$
W(G)=\sum_{\{u, v\} \subseteq V(G)} d(u, v),
$$

where $\{u, v\}$ runs over every pair of vertices in $G$.

Following this work, several versions of this index have been appearing since then, each one better adapted for its purpose. Let us mention, for example, the hyper-Wiener index, see [21,25] or the Harari index $[17,24]$.

A natural problem in the study of topological indices is, given some fixed parameters, to find the graphs that minimize (or maximize) their value on a certain set of graphs satisfying the restrictions given by the parameters (see e.g. $[1,2,4-8,14]$ ).

Herein, instead of doing this individually on each modified version of the Wiener index we consider a natural generalization of it which encompasses those mentioned above and try to work as generally as possible. In previous works we used the same strategy with trees, see [22]. Now, we are considering unicyclic graphs. A unicyclic graph is a graph containing exactly one cycle $[18$, p.41]. It is well known that if $G$ is a unicyclic graph with $n$ vertices, then $G$ has $n$ edges. 
The aim of this paper is to obtain new inequalities for a large family of topological indices restricted to unicyclic graphs, fixing or not the number of pendant vertices, and to characterize the extremal unicyclic graphs with respect to them. This problem, for other type of indices, was also addressed in [23].

Throughout this work, $G=(V(G), E(G))$ denotes a (non-oriented) finite connected simple (without multiple edges and loops) non-trivial $(E(G) \neq \emptyset)$ graph. Note that the connectivity of $G$ is not an important restriction, since every molecular graph is connected.

\section{Wiener index and its generalizations}

Motivated by the Wiener index, Randic introduced in [25] an extension of the Wiener index for trees, and this has come to be known as the hyperWiener index. In [21], this extension was generalized to graphs as

$$
W W(G)=\frac{1}{2} \sum_{\{u, v\} \subseteq V(G)} d(u, v)+\frac{1}{2} \sum_{\{u, v\} \subseteq V(G)} d(u, v)^{2} .
$$

$W W(G)$ has been useful in correlations (see, e.g., [12] and the references therein). For information about the hyper-Wiener index in mathematics see, e.g., [3], [12], [19].

Following [16] and [30], it is interesting to generalize the Wiener index in the following way

$$
W^{\lambda}(G)=\sum_{\{u, v\} \subseteq V(G)} d(u, v)^{\lambda}
$$

with $\lambda \in \mathbb{R}$. Obviously, if $\lambda=1$, then $W^{\lambda}$ coincides with the ordinary Wiener index $W$. Note that $W^{-2}$ is the Harary index; $W^{-1}$ is the reciprocal Wiener index; the quantity $W^{2}$ is closely related to the hyper-Wiener index, since $W W=\left(W^{1}+W^{2}\right) / 2$. Another topological index, proposed in [27] is expressed in terms of $W^{1}, W^{2}$ and $W^{3}$ as $\left(2 W^{1}+3 W^{2}+W^{3}\right) / 6$. See [20] for more connections of the same kind.

Three different variants of the $q$-Wiener index $(q>0, q \neq 1)$ were 
defined in [32] as

$$
\begin{aligned}
& W_{1}(G, q)=\sum_{\{u, v\} \subseteq V(G)}[d(u, v)]_{q}, \\
& W_{2}(G, q)=\sum_{\{u, v\} \subseteq V(G)}[d(u, v)]_{q} q^{L-d(u, v)}, \\
& W_{3}(G, q)=\sum_{\{u, v\} \subseteq V(G)}[d(u, v)]_{q} q^{d(u, v)},
\end{aligned}
$$

where $L$ is the diameter of $G$, and

$$
[k]_{q}=\frac{1-q^{k}}{1-q}=1+q+q^{2}+\cdots+q^{k-1} .
$$

Since $\lim _{q \rightarrow 1}[k]_{q}=k$, we have

$$
\lim _{q \rightarrow 1} W_{1}(G, q)=\lim _{q \rightarrow 1} W_{2}(G, q)=\lim _{q \rightarrow 1} W_{3}(G, q)=W(G) .
$$

Given any function $h: \mathbb{Z}^{+} \rightarrow \mathbb{R}$, let the $h$-Wiener index of $G$ be defined as

$$
W_{h}(G)=\sum_{\{u, v\} \subseteq V(G)} h(d(u, v)),
$$

A similar general index was introduced in [29] just for trees. This general approach allows to study in a unified way the previous indices.

If $P_{n}$ is the path graph with $n$ vertices, then

$$
W_{h}\left(P_{n}\right)=\sum_{1 \leq i<j \leq n} h(j-i)=\sum_{k=1}^{n-1}(n-k) h(k) .
$$

If $C_{n}$ is the cycle graph with $n$ vertices, then

- if $n$ is odd,

$$
W_{h}\left(C_{n}\right)=\sum_{j=1}^{\frac{n-1}{2}} n h(j)
$$


- if $n$ is even,

$$
W_{h}\left(C_{n}\right)=\sum_{j=1}^{\frac{n}{2}-1} n h(j)+\frac{n}{2} h\left(\frac{n}{2}\right)
$$

Given $n \geq 4$, let $J_{n}$ be the graph obtained by identifying a vertex from a cycle $C_{3}$ and the vertex with degree $n-3$ of a star graph with $n-2$ vertices, $S_{n-2}$. Then,

$$
W_{h}\left(J_{n}\right)=n h(1)+\frac{1}{2} n(n-3) h(2) .
$$

Given $3 \leq r \leq n$ and a function $h: \mathbb{Z}^{+} \rightarrow \mathbb{R}$, let us define the function $F_{h}(r, n)$ as follows:

- if $r$ is odd,

$$
F_{h}(r, n):=\sum_{j=1}^{\frac{r-1}{2}} r h(j)+\sum_{j=1}^{n-r}(n-r+1-j) h(j)+2 \sum_{k=1}^{n-r} \sum_{j=1}^{\frac{r-1}{2}} h(k+j),
$$

- if $r$ is even,

$$
\begin{aligned}
F_{h}(r, n):= & \sum_{j=1}^{\frac{r}{2}-1} r h(j)+\frac{r}{2} h\left(\frac{r}{2}\right)+\sum_{j=1}^{n-r}(n-r+1-j) h(j) \\
& +2 \sum_{k=1}^{n-r} \sum_{j=1}^{\frac{r}{2}-1} h(k+j)+\sum_{k=1}^{n-r} h\left(\frac{r}{2}+k\right) .
\end{aligned}
$$

Let $G_{r, n}$ be the graph obtained by identifying a vertex from a cycle $C_{r}$ and a vertex with degree 1 of a path graph $P_{n-r+1}$. Note that $G_{n, n}=C_{n}$.

As usual, if $a<b$, we use the convention

$$
\sum_{j=b}^{a} A(j)=0
$$

Proposition 1. Given $3 \leq r \leq n$ and a function $h: \mathbb{Z}^{+} \rightarrow \mathbb{R}$, then

$$
W_{h}\left(G_{r, n}\right)=F_{h}(r, n)
$$




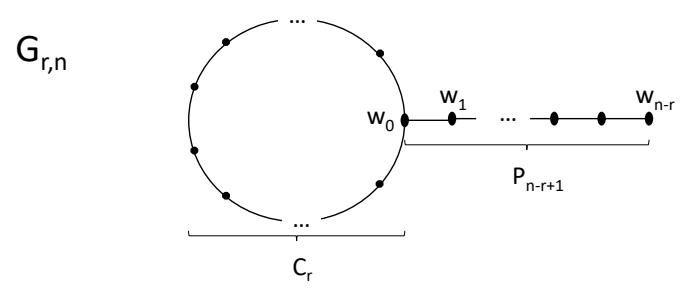

Figure 1. The graph $G_{r, n}$ is obtained by identifying a vertex from a cycle $C_{r}$ and a vertex with degree 1 of a path graph $P_{n-r+1}$.

Proof. Consider the graph $G_{r, n}$, let $w_{0}$ be the identified vertex which belongs to the cycle and the path and let $w_{1}, w_{2}, \ldots, w_{n-r}$ be the vertices in the path $P_{n-r+1}$ with $d\left(w_{0}, w_{k}\right)=k$. See Figure 1 .

Then,

$$
W_{h}\left(G_{r, n}\right)=W_{h}\left(C_{r}\right)+W_{h}\left(P_{n-r+1}\right)+\sum_{w_{0} \neq v \in C_{r}, w_{0} \neq w_{i} \in P_{n-r+1}} h\left(d\left(v, w_{i}\right)\right) .
$$

Suppose $r$ is odd. Then, notice that for every $1 \leq k \leq n-r$,

$$
\sum_{w_{0} \neq v \in C_{r}} h\left(d\left(v, w_{k}\right)\right)=2 \sum_{j=1}^{\frac{r-1}{2}} h(j+k)
$$

Thus,

$$
W_{h}\left(G_{r, n}\right)=\sum_{j=1}^{\frac{r-1}{2}} r h(j)+\sum_{j=1}^{n-r}(n-r+1-j) h(j)+2 \sum_{k=1}^{n-r} \sum_{j=1}^{\frac{r-1}{2}} h(k+j) .
$$

In particular, the following expression of $F_{h}(3, n)$ will be useful below in Lemma 1.

$$
F_{h}(3, n)=3 h(1)+\sum_{j=1}^{n-3}(n-2-j) h(j)+2 \sum_{k=1}^{n-3} h(k+1)=n h(1)+\sum_{j=2}^{n-2}(n-j) h(j) .
$$


Suppose $r$ is even. Then, notice that for every $1 \leq k \leq n-r$,

$$
\sum_{w_{0} \neq v \in C_{r}} h\left(d\left(v, w_{k}\right)\right)=2 \sum_{j=1}^{\frac{r}{2}-1} h(j+k)+h\left(\frac{r}{2}+k\right) .
$$

Thus,

$$
\begin{aligned}
W_{h}\left(G_{r, n}\right)= & \sum_{j=1}^{\frac{r}{2}-1} r h(j)+\frac{r}{2} h\left(\frac{r}{2}\right)+\sum_{j=1}^{n-r}(n-r+1-j) h(j) \\
& +2 \sum_{k=1}^{n-r} \sum_{j=1}^{\frac{r}{2}-1} h(k+j)+\sum_{k=1}^{n-r} h\left(\frac{r}{2}+k\right) .
\end{aligned}
$$

Remark. Since any graph with $n$ vertices has $\frac{1}{2} n(n-1)$ pairs of vertices, we have that

- If $r$ is odd,

$$
\frac{1}{2} n(n-1)=n+\sum_{j=2}^{n-2}(n-j)=\sum_{j=1}^{\frac{r-1}{2}} r+\sum_{j=1}^{n-r}(n-r+1-j)+2 \sum_{k=1}^{n-r} \sum_{j=1}^{\frac{r-1}{2}} 1 .
$$

- If $r$ is even,

$$
\begin{aligned}
\frac{1}{2} n(n-1) & =n+\sum_{j=2}^{n-2}(n-j) \\
& =\sum_{j=1}^{\frac{r}{2}-1} r+\frac{r}{2}+\sum_{j=1}^{n-r}(n-r+1-j)+2 \sum_{k=1}^{n-r} \sum_{j=1}^{\frac{r}{2}-1} 1+\sum_{k=1}^{n-r} 1 .
\end{aligned}
$$

Lemma 1. Given $4 \leq r \leq n$ with $r$ even and a strictly increasing function $h: \mathbb{Z}^{+} \rightarrow \mathbb{R}$, then $F_{h}(3, n)>F_{h}(r, n)$. If $h$ is a strictly decreasing function, then $F_{h}(3, n)<F_{h}(r, n)$.

Proof. First of all, note that the second statement is a consequence of the first one, if we consider the function $-h$. Hence, we can assume that $h$ is 
a strictly increasing function. We have

$$
\begin{aligned}
& F_{h}(3, n)>F_{h}(r, n) \\
& \Leftrightarrow \sum_{j=2}^{n-r}(n-j) h(j)+\sum_{j=n-r+1}^{n-2}(n-j) h(j) \\
& \quad>\sum_{j=2}^{\frac{r}{2}-1} r h(j)+\frac{r}{2} h\left(\frac{r}{2}\right)+\sum_{j=\frac{r}{2}+1}^{n-\frac{r}{2}} h(j)+\sum_{j=2}^{n-r}(n-r+1-j) h(j) \\
& \quad+2 \sum_{k=1}^{n-r} \sum_{j=1}^{\frac{r}{2}-1} h(k+j) .
\end{aligned}
$$

Case 1. Suppose $n-r<\frac{r}{2}-1$. Then,

$$
\begin{aligned}
& F_{h}(3, n)>F_{h}(r, n) \\
& \Leftrightarrow \quad \sum_{j=n-r+1}^{n-2}(n-j) h(j) \\
& \quad>\sum_{j=2}^{n-r} h(j)+\sum_{j=n-r+1}^{\frac{r}{2}-1} r h(j)+\frac{r}{2} h\left(\frac{r}{2}\right)+\sum_{j=\frac{r}{2}+1}^{n-\frac{r}{2}} h(j)+2 \sum_{k=1}^{n-r} \sum_{j=1}^{\frac{r}{2}-1} h(k+j) \\
& \Leftrightarrow \quad(n-r) h\left(\frac{r}{2}\right)+\sum_{j=\frac{r}{2}+1}^{n-\frac{r}{2}}(n-j-1) h(j)+\sum_{j=n-\frac{r}{2}+1}^{n-2}(n-j) h(j) \\
& \quad>\sum_{j=2}^{n-r} h(j)+\sum_{j=n-r+1}^{\frac{r}{2}-1}(r-n+j) h(j)+2 \sum_{k=1}^{n-r} \sum_{j=1}^{\frac{r}{2}-1} h(k+j) .
\end{aligned}
$$

Since $n-r<\frac{r}{2}-1$,

$$
\begin{aligned}
& 2 \sum_{k=1}^{n-r} \sum_{j=1}^{\frac{r}{2}-1} h(k+j)=2 h(2)+4 h(3)+\cdots+2(n-r) h(n-r+1)+\cdots \\
& \quad+2(n-r) h\left(\frac{r}{2}\right)+2(n-r-1) h\left(\frac{r}{2}+1\right)+\cdots+2 h\left(n-\frac{r}{2}-1\right) .
\end{aligned}
$$

Let

$$
M=(n-r-1)+\frac{1}{2}\left(\frac{3 r}{2}-n-1\right)\left(\frac{3 r}{2}-n\right)+(n-r)(2 r-n-1) .
$$


Then, since $h$ is strictly increasing, it follows that

$$
\begin{aligned}
\sum_{j=2}^{n-r} h(j)+ & \sum_{j=n-r+1}^{\frac{r}{2}-1}(r-n+j) h(j)+2 \sum_{k=1}^{n-r} \sum_{j=1}^{\frac{r}{2}-1} h(k+j) \\
& <M h\left(\frac{r}{2}\right)+2(n-r-1) h\left(\frac{r}{2}+1\right)+\cdots+2 h\left(n-\frac{r}{2}-1\right) \\
& =M h\left(\frac{r}{2}\right)+\sum_{j=\frac{r}{2}+1}^{n-\frac{r}{2}-1} 2\left(n-\frac{r}{2}-j\right) h(j) .
\end{aligned}
$$

Therefore, since in

$$
(n-r) h\left(\frac{r}{2}\right)+\sum_{j=\frac{r}{2}+1}^{n-\frac{r}{2}}(n-j-1) h(j)+\sum_{j=n-\frac{r}{2}+1}^{n-2}(n-j) h(j)
$$

the function $h$ is evaluated always in numbers greater or equal than $\frac{r}{2}$ and Remark 2 gives

$$
\begin{aligned}
(n-r)+\sum_{j=\frac{r}{2}+1}^{n-\frac{r}{2}}(n-j-1) & +\sum_{j=n-\frac{r}{2}+1}^{n-2}(n-j) \\
& =\sum_{j=2}^{n-r} 1+\sum_{j=n-r+1}^{\frac{r}{2}-1}(r-n+j)+2 \sum_{k=1}^{n-r} \sum_{j=1}^{\frac{r}{2}-1} 1
\end{aligned}
$$

it suffices to check that

$$
\sum_{j=\frac{r}{2}+1}^{n-\frac{r}{2}-1} 2\left(n-\frac{r}{2}-j\right) h(j)<\sum_{j=\frac{r}{2}+1}^{n-\frac{r}{2}-1}(n-j-1) h(j) .
$$

Hence, it suffices to show for every $\frac{r}{2}+1 \leq j \leq n-\frac{r}{2}-1$ that

$$
2 n-r-2 j<n-j-1,
$$

and this is equivalent to $n-r<j-1$, which follows from

$$
j-1 \geq \frac{r}{2}>\frac{r}{2}-1>n-r
$$


This finishes the proof of Case 1 .

Case 2. Suppose $\frac{r}{2}-1=n-r$. Then,

$$
\begin{aligned}
& F_{h}(3, n)>F_{h}(r, n) \\
& \Leftrightarrow \quad \sum_{j=2}^{\frac{r}{2}-1}\left(\frac{3 r}{2}-1-j\right) h(j)+\sum_{j=\frac{r}{2}}^{\frac{3 r}{2}-3}\left(\frac{3 r}{2}-1-j\right) h(j) \\
& \quad>\sum_{j=2}^{\frac{r}{2}-1}\left(\frac{3 r}{2}-j\right) h(j)+\frac{r}{2} h\left(\frac{r}{2}\right)+\sum_{j=\frac{r}{2}+1}^{r-1} h(j)+2 \sum_{k=1}^{\frac{r}{2}-1} \sum_{j=1}^{\frac{r}{2}-1} h(k+j) \\
& \Leftrightarrow \quad \sum_{j=\frac{r}{2}}^{\frac{3 r}{2}-3}\left(\frac{3 r}{2}-1-j\right) h(j) \\
& \quad>\sum_{j=2}^{\frac{r}{2}-1} h(j)+\frac{r}{2} h\left(\frac{r}{2}\right)+\sum_{j=\frac{r}{2}+1}^{r-1} h(j)+2 \sum_{k=1}^{\frac{r}{2}-1} \sum_{j=1}^{\frac{r}{2}-1} h(k+j) .
\end{aligned}
$$

Notice that

$$
\begin{aligned}
2 \sum_{k=1}^{\frac{r}{2}-1} \sum_{j=1}^{\frac{r}{2}-1} h(k+j) & =2 h(2)+4 h(3)+\cdots+2\left(\frac{r}{2}-1\right) h\left(\frac{r}{2}\right) \\
& +2\left(\frac{r}{2}-2\right) h\left(\frac{r}{2}+1\right)+\cdots+2 h(r-2) \\
& =\sum_{j=2}^{\frac{r}{2}} 2(j-1) h(j)+\sum_{j=\frac{r}{2}+1}^{r-2} 2(r-1-j) h(j) .
\end{aligned}
$$

Since $h$ is strictly increasing, it follows that

$$
\begin{aligned}
& \sum_{j=2}^{\frac{r}{2}-1} h(j)+\frac{r}{2} h\left(\frac{r}{2}\right)+\sum_{j=\frac{r}{2}+1}^{r-1} h(j)+2 \sum_{k=1}^{\frac{r}{2}-1} \sum_{j=1}^{\frac{r}{2}-1} h(k+j) \\
& \quad<\left(\frac{r}{2}-1\right)\left(\frac{r}{2}+2\right) h\left(\frac{r}{2}\right)+\sum_{j=\frac{r}{2}+1}^{r-1} h(j)+\sum_{j=\frac{r}{2}+1}^{r-2} 2(r-1-j) h(j) .
\end{aligned}
$$

Thus, it suffices to check that for every $\frac{r}{2}+1 \leq j$ we have $2 r-1-2 j<$ $\frac{3 r}{2}-1-j$, which is direct. 
Case 3. Suppose $\frac{r}{2}-1<n-r$. Then,

$$
\begin{aligned}
& F_{h}(3, n)>F_{h}(r, n) \\
& \Leftrightarrow \quad \sum_{j=2}^{n-r}(r-1) h(j)+\sum_{j=n-r+1}^{n-2}(n-j) h(j) \\
& >\sum_{j=2}^{\frac{r}{2}-1} r h(j)+\frac{r}{2} h\left(\frac{r}{2}\right)+\sum_{j=\frac{r}{2}+1}^{n-\frac{r}{2}} h(j)+2 \sum_{k=1}^{n-r} \sum_{j=1}^{\frac{r}{2}-1} h(k+j) \\
& \Leftrightarrow \quad\left(\frac{r}{2}-1\right) h\left(\frac{r}{2}\right)+\sum_{j=\frac{r}{2}+1}^{n-r}(r-2) h(j)+\sum_{j=n-r+1}^{n-2}(n-j) h(j) \\
& >\sum_{j=2}^{n-\frac{r}{2}} h(j)+\sum_{j=n-r+1}^{\frac{r}{2}-1} h(j)+2 \sum_{k=1}^{n-r} \sum_{j=1}^{\frac{r}{2}-1} h(k+j) .
\end{aligned}
$$

Since $\frac{r}{2}-1<n-r$,

$$
\begin{aligned}
& 2 \sum_{k=1}^{n-r} \sum_{j=1}^{\frac{r}{2}-1} h(k+j)=2 h(2)+4 h(3)+\cdots+2\left(\frac{r}{2}-1\right) h\left(\frac{r}{2}\right)+\cdots \\
& \quad+2\left(\frac{r}{2}-1\right) h(n-r+1)+2\left(\frac{r}{2}-2\right) h(n-r+2)+\cdots+2 h\left(n-\frac{r}{2}-1\right) \\
& \quad=\sum_{j=2}^{\frac{r}{2}} 2(j-1) h(j)+\sum_{j=\frac{r}{2}+1}^{n-r}(r-2) h(j)+\sum_{j=n-r+1}^{n-\frac{r}{2}-1}(2 n-r-2 j) h(j) .
\end{aligned}
$$

Since $h$ is strictly increasing, it follows that

$$
\begin{aligned}
\sum_{j=2}^{\frac{r}{2}-1} h(j) & +\sum_{j=n-r+1}^{n-\frac{r}{2}} h(j)+2 \sum_{k=1}^{n-r} \sum_{j=1}^{\frac{r}{2}-1} h(k+j) \\
& <\left(\frac{r^{2}}{4}-2\right) h\left(\frac{r}{2}\right)+\sum_{j=n-r+1}^{n-\frac{r}{2}} h(j)+\sum_{j=\frac{r}{2}+1}^{n-r}(r-2) h(j) \\
& +\sum_{j=n-r+1}^{n-\frac{r}{2}-1}(2 n-r-2 j) h(j) .
\end{aligned}
$$


Therefore, since in

$$
\left(\frac{r}{2}-1\right) h\left(\frac{r}{2}\right)+\sum_{j=\frac{r}{2}+1}^{n-r}(r-2) h(j)+\sum_{j=n-r+1}^{n-2}(n-j) h(j)
$$

the function $h$ is evaluated always in numbers greater or equal than $\frac{r}{2}$, it suffices to check that for every $n-r+1 \leq j$ we have $2 n-r-2 j+1 \leq n-j$, which is immediate.

Lemma 2. Given $5 \leq r \leq n$ with $r$ odd and a strictly increasing function $h: \mathbb{Z}^{+} \rightarrow \mathbb{R}$, then $F_{h}(3, n)>F_{h}(r, n)$. If $h$ is a strictly decreasing function, then $F_{h}(3, n)<F_{h}(r, n)$.

Proof. The second statement is a consequence of the first one, if we consider the function $-h$. Therefore, we can assume that $h$ is a strictly increasing function.

Let us recall that

$$
F_{h}(3, n)=n h(1)+\sum_{j=2}^{n-2}(n-j) h(j) .
$$

Case 1. Suppose $n-r<\frac{r-1}{2}$. Then,

$$
F_{h}(r, n)=\sum_{j=1}^{n-r}(n+1-j) h(j)+\sum_{j=n-r+1}^{\frac{r-1}{2}} r h(j)+2 \sum_{k=1}^{n-r} \sum_{j=1}^{\frac{r-1}{2}} h(k+j) .
$$

Therefore,

$$
\begin{aligned}
& F_{h}(3, n)>F_{h}(r, n) \\
& \Leftrightarrow \sum_{j=n-r+1}^{n-2}(n-j) h(j)>\sum_{j=2}^{n-r} h(j)+\sum_{j=n-r+1}^{\frac{r-1}{2}} r h(j)+2 \sum_{k=1}^{n-r} \sum_{j=1}^{\frac{r-1}{2}} h(k+j) \\
& \Leftrightarrow \sum_{j=\frac{r+1}{2}}^{n-2}(n-j) h(j)>\sum_{j=2}^{n-r} h(j)+\sum_{j=n-r+1}^{\frac{r-1}{2}}(j-n+r) h(j)+2 \sum_{k=1}^{n-r} \sum_{j=1}^{\frac{r-1}{2}} h(k+j) .
\end{aligned}
$$


Since $n-r<\frac{r-1}{2}$,

$$
\begin{aligned}
2 \sum_{k=1}^{n-r} \sum_{j=1}^{\frac{r-1}{2}} h(k+j)= & 2 h(2)+\cdots+2(n-r) h(n-r+1)+\cdots \\
& +2(n-r) h\left(\frac{r+1}{2}\right)+\cdots+2 h\left(n-\frac{r}{2}-\frac{1}{2}\right) .
\end{aligned}
$$

Let

$$
\begin{aligned}
N= & n-r-1+\frac{1}{2} \frac{(3 r-2 n-1)}{2} \frac{(3 r-2 n+1)}{2} \\
& +(n-r)(n-r+1)+(3 r-2 n-1)(n-r) .
\end{aligned}
$$

Therefore, since $h$ is strictly increasing,

$$
\begin{gathered}
\sum_{j=2}^{n-r} h(j)+\sum_{j=n-r+1}^{\frac{r-1}{2}}(j-n+r) h(j)+2 \sum_{k=1}^{n-r} \sum_{j=1}^{\frac{r-1}{2}} h(k+j) \\
<N h\left(\frac{r+1}{2}\right)+\sum_{j=\frac{r+3}{2}}^{n-\frac{r}{2}-\frac{1}{2}} 2\left(n-\frac{r}{2}+\frac{1}{2}-j\right) h(j) .
\end{gathered}
$$

Thus, it suffices to check that for every $\frac{r+3}{2} \leq j \leq n-\frac{r}{2}-\frac{1}{2}$ we have

$$
2 n-r+1-2 j<n-j,
$$

and this is equivalent to $n-r<j-1$, which is immediate since

$$
j-1 \geq \frac{r+1}{2}>n-r .
$$


Case 2. Suppose $\frac{r-1}{2} \leq n-r$. Then,

$$
\begin{aligned}
F_{h}(r, n) & =\sum_{j=1}^{\frac{r-1}{2}} r h(j)+\sum_{j=1}^{\frac{r-1}{2}}(n-r+1-j) h(j)+\sum_{j=\frac{r+1}{2}}^{n-r}(n-r+1-j) h(j) \\
& +2 \sum_{k=1}^{n-r} \sum_{j=1}^{\frac{r-1}{2}} h(k+j) \\
& =\sum_{j=1}^{\frac{r-1}{2}}(n+1-j) h(j)+\sum_{j=\frac{r+1}{2}}^{n-r}(n-r+1-j) h(j)+2 \sum_{k=1}^{n-r} \sum_{j=1}^{\frac{r-1}{2}} h(k+j) .
\end{aligned}
$$

Therefore,

$$
\begin{aligned}
& F_{h}(3, n)>F_{h}(r, n) \\
& \Leftrightarrow \sum_{j=\frac{r+1}{2}}^{n-2}(n-j) h(j)>\sum_{j=2}^{\frac{r-1}{2}} h(j)+\sum_{j=\frac{r+1}{2}}^{n-r}(n-r+1-j) h(j)+2 \sum_{k=1}^{n-r} \sum_{j=1}^{\frac{r-1}{2}} h(k+j) \\
& \Leftrightarrow \sum_{j=\frac{r+1}{2}}^{n-r}(r-1) h(j)+\sum_{j=n-r+1}^{n-2}(n-j) h(j)>\sum_{j=2}^{\frac{r-1}{2}} h(j)+2 \sum_{k=1}^{n-r} \sum_{j=1}^{\frac{r-1}{2}} h(k+j) .
\end{aligned}
$$

Since $\frac{r-1}{2} \leq n-r$

$$
\begin{aligned}
2 \sum_{k=1}^{n-r} \sum_{j=1}^{\frac{r-1}{2}} h(k+j)= & 2 h(2)+\cdots+2\left(\frac{r-1}{2}\right) h\left(\frac{r+1}{2}\right)+\cdots \\
& +2\left(\frac{r-1}{2}\right) h(n-r+1)+\cdots+2 h\left(n-\frac{r}{2}-\frac{1}{2}\right) .
\end{aligned}
$$

Let $N^{\prime}=\frac{r^{2}+2 r-7}{4}$. Therefore, since $h$ is strictly increasing,

$$
\begin{aligned}
\sum_{j=2}^{\frac{r-1}{2}} h(j)+2 \sum_{k=1}^{n-r} \sum_{j=1}^{\frac{r-1}{2}} h(k+j) & <N^{\prime} h\left(\frac{r+1}{2}\right)+\sum_{j=\frac{r+3}{2}}^{n-r}(r-2) h(j) \\
& +\sum_{j=n-r+1}^{n-\frac{r}{2}-\frac{1}{2}}(2 n-r+1-2 j) h(j) .
\end{aligned}
$$

Thus, it suffices to check that for every $\frac{r+3}{2} \leq j \leq n-r$ we have $r-2 \leq r-1$, 
and for every $n-r+1 \leq j \leq n-\frac{r}{2}-\frac{1}{2}$ we have

$$
2 n-r+1-2 j \leq n-j \quad \Leftrightarrow \quad n-r+1 \leq j,
$$

which is trivial.

Remark. Notice that given $3<r \leq n$ and a strictly increasing function $h: \mathbb{Z}^{+} \rightarrow \mathbb{R}$, although $F_{h}(3, n)>F_{h}(r, n), F_{h}(r, n)$ is not necessarily decreasing on $r$. For example, suppose $h$ is the identity map. Then

$$
F_{h}(12,13)=13 h(1)+14(h(2)+h(3)+h(4)+h(5))+8 h(6)+h(7)
$$

and

$$
F_{h}(11,13)=13 h(1)+14 h(2)+15(h(3)+h(4)+h(5))+4 h(6)+2 h(7) .
$$

Thus,

$$
\begin{aligned}
F_{h}(12,13)-F_{h}(11,13) & =4 h(6)-h(3)-h(4)-h(5)-h(7) \\
& =24-3-4-5-7=5>0
\end{aligned}
$$

and $F_{h}(12,13)>F_{h}(11,13)$.

Let us recall the following definitions from [10]. A vertex of degree at least three in a graph $G$ will be called a major vertex of $G$. Any end-vertex (a vertex of degree one) $u$ of $G$ is said to be a terminal vertex of a major vertex $v$ of $G$ if $d_{G}(u, v)<d_{G}(u, w)$ for every other major vertex $w$ of $G$. The terminal degree of a major vertex $v$ is the number of terminal vertices of $v$. A major vertex $v$ of $G$ is called exterior major vertex if it has nonzero terminal degree. Let $\mathcal{M}(G)$ be the set of exterior major vertices of $G$ having terminal degree greater than one.

The following result is elementary.

Lemma 3. If $T$ is a tree, then $\mathcal{M}(T)=\emptyset$ if and only if $T$ is a path graph.

Given a unicyclic graph $G$ and a vertex $v$ let us denote $G_{v}$ the connected component of $G \backslash v$ intersecting the cycle. Then, the complement $T_{v}:=$ $G \backslash G_{v}$ is a tree (possibly being the the single vertex $v$ ). 
Theorem 2. Let $G$ be a unicyclic graph with $n \geq 6$ vertices.

(1) If $h$ is a strictly increasing function, then

$$
n h(1)+\frac{1}{2} n(n-3) h(2) \leq W_{h}(G) \leq F_{h}(3, n),
$$

the lower bound is attained if and only if $G=J_{n}$ and the upper bound is attained if and only if $G=G_{3, n}$.

(2) If $h$ is a strictly decreasing function, then

$$
F_{h}(3, n) \leq W_{h}(G) \leq n h(1)+\frac{1}{2} n(n-3) h(2),
$$

the lower bound is attained if and only if $G=G_{3, n}$ and the upper bound is attained if and only if $G=J_{n}$.

Proof. The second statement is a consequence of the first one, if we consider the function $-h$. Hence, we can assume that $h$ is a strictly increasing function.

Since $G$ is a unicyclic graph with $n$ vertices, there are $n$ edges and $n$ pairs of adjacent vertices. Thus, there are $\left(\begin{array}{l}n \\ 2\end{array}\right)-n=\frac{1}{2} n(n-3)$ pairs of vertices at distance at least 2 . The lower bound is attained if and only if $T$ has diameter 2. Notice that this means that the cycle has at most 5 vertices and since $n \geq 6$ and any vertex which is not in the cycle is at distance at most 2 from any vertex in it, the cycle is necessarily $C_{3}$. Therefore, the lower bound is attained if and only if $G=J_{n}$.

Assume that $G$ is a unicyclic graph with $n$ vertices such that $W_{h}(G)$ is maximal and let $C$ be the cycle.

Seeking for a contradiction, consider any vertex $v \in G$ such that $v$ is not in the unique cycle and $\operatorname{deg}(v) \geq 3$ or $v$ is in the cycle and $\operatorname{deg}(v) \geq 4$. Then, there is an exterior major vertex $w$ in $T_{v}$ (where possibly $v=w$ ). Hence, consider two terminal vertices of $w, u_{1}, u_{2}$ with $d\left(w, u_{1}\right)=k$. Let us define new vertices $\left\{v_{1}, \ldots, v_{k}\right\}$ and let

$$
G^{\prime}:=\left(G \backslash\left[w, u_{1}\right]\right) \cup u_{2} v_{1} \cup v_{1} v_{2} \cup \cdots \cup v_{k-1} v_{k} .
$$

Thus, it is immediate to see that, since $h$ is strictly increasing, $W_{h}\left(G^{\prime}\right)>$ 
$W_{h}(G)$ leading to contradiction. Therefore, $\operatorname{deg}(v) \leq 2$ for every vertex $v \notin C$, and $\operatorname{deg}(v) \leq 3$ for every vertex $v \in C$.

Suppose there exist $v_{1}, v_{2} \in C$ with $\operatorname{deg}\left(v_{i}\right)=3$ for $i=1,2$. Then, $T_{v_{i}}$ is a path $\left[v_{i}, u_{i}\right]$ of length $d\left(\left[v_{i}, u_{i}\right]\right)=l_{i}$. Let $D_{i}:=\sum_{v \in G \backslash\left\{T_{v_{1}} \cup T_{v_{2}}\right\}} d\left(v_{i}, v\right)$ for $i=1,2$. If $D_{i}<D_{j}$ and $l_{i}>l_{j}$, consider the vertex $w \in\left[v_{i}, u_{i}\right]$ such that $d\left(v_{i}, w\right)=l_{j}$, let $s=l_{i}-l_{j}$ and let us define new vertices $\left\{w_{1}, \ldots, w_{s}\right\}$. Then, let

$$
G^{\prime}:=\left(G \backslash\left[w, u_{i}\right]\right) \cup u_{j} w_{1} \cup w_{1} w_{2} \cup \cdots \cup w_{s-1} w_{s} .
$$

Since $D_{i}<D_{j}$ and $h$ is strictly increasing, it follows that $W_{h}\left(G^{\prime}\right)>W_{h}(G)$ leading to contradiction. Thus, we may assume, relabeling if necessary, that $D_{1} \leq D_{2}$ and $l_{1} \leq l_{2}$. Hence, let us define new vertices $\left\{a_{1}, \ldots, a_{l_{1}}\right\}$ and let

$$
G^{\prime}:=\left(G \backslash\left[v_{1}, u_{1}\right]\right) \cup u_{2} a_{1} \cup a_{1} a_{2} \cup \cdots \cup a_{l_{1}-1} a_{l_{1}} .
$$

Since $D_{1} \leq D_{2}, l_{1} \leq l_{2}$ and $h$ is strictly increasing, it follows that $W_{h}\left(G^{\prime}\right)>$ $W_{h}(G)$ leading to contradiction.

Therefore, $G$ is a unicyclic graph with at most one vertex with degree 3 and $G=G_{r, n}$ where $r$ is the length of the cycle. By lemmas 1 and 2, $G=G_{3, n}$.

Corollary. If $G$ is a unicyclic graph with $n \geq 6$ vertices, then

$$
n(n-2) \leq W(G) \leq 1+\sum_{k=1}^{n-2}(n-k) k=\frac{n^{3}-7 n+12}{6},
$$

the lower bound is attained if and only if $G=J_{n}$ and the upper bound is attained if and only if $G=G_{3, n}$. 
Acknowledgment: The first author was partially supported by a grant from Ministerio de Ciencia, Innovación y Universidades (PGC2018-098321B-I00), Spain; the second author was partially supported by a grant from Agencia Estatal de Investigación (PID2019-106433GB-I00/AEI/10.13039 /501100011033), Spain, and by the Madrid Government (Comunidad de Madrid-Spain) under the Multiannual Agreement with UC3M in the line of Excellence of University Professors (EPUC3M23), and in the context of the V PRICIT (Regional Programme of Research and Technological Innovation).

\section{References}

[1] B. Bollobás, P. Erdös, Graphs of extremal weights, Ars Comb. 50 (1998) 225-233.

[2] B. Bollobás, P. Erdös, A. Sarkar, Extremal graphs for weights, Discr. Math. 200 (1999) 5-19.

[3] G. G. Cash, Relationship between the Hosoya polynomial and the hyper-Wiener index, Appl. Math. Lett. 15 (2002) 893-895.

[4] R. Cruz, H. Giraldo, J. Rada, Extremal values of vertex-degree topological indices over hexagonal systems, MATCH Commun. Math. Comput. Chem. 70 (2013) 501-512.

[5] K. C. Das, Maximizing the sum of the squares of the degrees of a graph, Discr. Math. 285 (2004) 57-66.

[6] Z. Du, B. Zhou, N. Trinajstić, Minimum general sum-connectivity index of unicyclic graphs, J. Math. Chem. 48 (2010) 697-703.

[7] Z. Du, B. Zhou, N. Trinajstić, Minimum sum-connectivity indices of trees and unicyclic graphs of a given matching number, J. Math. Chem. 47 (2010) 842-855.

[8] C. S. Edwards, The largest vertex degree sum for a triangle in a graph, Bull. London Math. Soc. 9 (1977) 203-208.

[9] E. Estrada, L. Torres, L. Rodríguez, I. Gutman, An atom-bond connectivity index: Modelling the enthalpy of formation of alkanes, Indian J. Chem. 37A (1998) 849-855.

[10] A. Estrada-Moreno, J. A. Rodríguez-Velázquez, I. G. Yero, The $k$ metric dimension of a graph, Appl. Math. Inform. Sci. 9 (2015) 2829 2840 . 
[11] I. Gutman, Distance in thorny graph. Publ. Inst. Math. (Beograd) 63 (1998) 31-36.

[12] I. Gutman, Relation between hyper-Wiener and Wiener index, Chem. Phys. Lett. 364 (2002) 352-356.

[13] I. Gutman, B. Furtula, Vertex-degree-based molecular structure descriptors of benzenoid systems and phenylenes, J. Serb. Chem. Soc. 77 (2012) 1031-1036.

[14] I. Gutman, B. Furtula, M. Ivanović, Notes on trees with minimal atom-bond connectivity index, MATCH Commun. Math. Comput. Chem. 67 (2012) 467-482.

[15] I. Gutman, J. Tošović, Testing the quality of molecular structure descriptors. Vertex-degree-based topological indices, J. Serb. Chem. Soc. 78 (2013) 805-810.

[16] I. Gutman, D. Vukičević, J. Žerovnik, A class of modified Wiener indices. Croat. Chem. Acta 77 (2004) 103-109.

[17] O. Ivanciuc, T. S. Balaban, A. T. Balaban, Reciprocal distance matrix, related local vertex invariants and topological indices, J. Math. Chem. 12 (1993) 309-318.

[18] F. Harary, Graph Theory, Addison-Wesley, Reading, 1994.

[19] S. Klavžar, P. Žigert, I. Gutman, An algorithm for the calculation of the hyper-Wiener index of benzenoid hydrocarbons, Comput. Chem. 24 (2000) 229-233.

[20] D. J. Klein, I. Gutman, Wiener-number-related sequences, J. Chem. Inf. Comput. Sci. 39 (1999) 534-536.

[21] D. J. Klein, I. Lukovits, I. Gutman, On the definition of the hyperWiener index for cycle-containing structures, J. Chem. Inf. Comput. Sci. 35 (1995) 50-52.

[22] A. Martínez-Pérez, J. M. Rodríguez, A unified approach to bounds for topological indices on trees and applications, MATCH Commun. Math. Comput. Chem. 82 (2019) 679-698.

[23] A. Martínez-Pérez, J. M. Rodríguez, Upper and lower bounds for topological indices on unicyclic graphs, submitted.

[24] D. Plavšić, S. Nikolić, N. Trinajstić, Z. Mihalić, On the Harary index for the characterization of chemical graphs, J. Math. Chem. 12 (1993) $235-250$. 
[25] M. Randić, Novel molecular descriptor for structure-property studies, Chem. Phys. Lett. 211 (1993) 478-483.

[26] M. Randić, D. Plavšić, N. Lerš, Variable connectivity index for cyclecontaining structures, J. Chem. Inf. Comput. Sci. 41 (2001) 657-662.

[27] S. S. Tratch, M. I. Stankevich, N. S. Zefirov, Combinatorial models and algorithms in chemistry. The expanded Wiener numbers - A novel topological index, J. Comput. Chem. 11 (1990) 899-908.

[28] M. Vöge, A. J. Guttmann, I. Jensen, On the number of benzenoid hydrocarbons, J. Chem. Inf. Comput. Sci. 42 (2002) 456-466.

[29] D. Vukičević, J. Sedlar, On indices of Wiener and anti-Wiener type, Discr. Appl. Math. 251 (2018) 290-298.

[30] D. Vukičević, J. Žerovnik, Variable Wiener indices, MATCH Commun. Math. Comput. Chem. 53 (2005) 385-402.

[31] H. Wiener, Structural determination of paraffin boiling points, J. Am. Chem. Soc. 69 (1947) 17-20.

[32] Y. S. Zhang, I. Gutman, J. G. Liu, Z. C. Mu, q-Analog of Wiener index, MATCH Commun. Math. Comput. Chem. 67 (2012) 347-356.

[33] S. Zhang, W. Wang, T. C. E. Cheng, Bicyclic graphs with the first three smallest and largest values of the first general Zagreb index, MATCH Commun. Math. Comput. Chem. 55 (2006) 579-592. 